\title{
Integration of Non-volatile Ferroelectric Actuators in Silicon Photonics Circuits
}

\author{
Isis Maqueira-Albo, Sara Varotto*, Marco Asa*, Christian Rinaldi*, Matteo Cantoni*, Riccardo Bertacco*, \\ Francesco Morichetti \\ Dipartimento di Elettronica, Informazione e Bioingegneria Politecnico di Milano, 20133 Milano, Italy \\ *Dipartimento di Fisica, Politecnico di Milano, 20133 Milano, Italy \\ Tel: (+39) 0223998954, Fax: (+39)0223993413, e-mail: francesco.morichetti@polimi.it
}

\begin{abstract}
In this work, we investigate a novel approach to realize non-volatile phase-actuators integrated in silicon photonic waveguides. We aim to exploit the ferroelectric response of polycrystalline $\mathrm{BaTiO}_{3}$ (poly-BTO) grown by pulsed laser deposition (PLD), whose domain can be re-oriented by applying an external electric field so as to induce a large change of the refractive index. Owing to the polarization remanence of ferroelectric materials, such refractive index variation is partially maintained when the electric field is turned off. Experimental results on poly-BTO demonstrate the possibility to achieve a non-volatile change of the poly-BTO refractive index in the order of $10^{-2}$, which is consistent with a $90^{\circ}$ reorientation of the ferroelectric domains. We also integrated thin poly-BTO films in $\mathrm{Si}$ waveguides with a low additional propagation loss $(<1 \mathrm{~dB} / \mathrm{mm})$, enabling the realization of photonic integrated circuits (PICs) with the proposed poly-BTO Si-photonics platform.
\end{abstract}

Keywords: Integrated optics materials, ferroelectric, optical waveguides, photonic integrated circuits, silicon photonics.

\section{INTRODUCTION}

Low-energy optical actuators are needed in integrated optics to either compensate fabrication tolerances or dynamically control and calibrate the functionality of reconfigurable and programmable circuits. Conventional actuators technologies exploit local heating of the optical waveguides as well as carrier injection/depletion effects in semiconductor waveguides. Albeit being effective and well consolidated, these solutions typically require high power consumption or suffer from large crosstalk that limits PIC scalability. Non-volatile actuators capable to maintain the switching state without an applied control signal are a promising route to tackle this issue. To this aim, several approaches based on phase change materials (GST) [1] and insulator-metal phase transition materials [2] have been proposed, which however mainly provide a control of the imaginary part of the refractive index of the waveguide, thus realizing "intensity actuators" solutions only.

In this work, we propose a novel approach to realize self-holding phase actuators integrated in Si waveguides. We aim to exploit the ferroelectric response of polycrystalline $\mathrm{BaTiO}_{3}$ (poly-BTO) directly deposited on a $\mathrm{Si}$ substrate. We observed a large non-volatile change of the poly-BTO refractive index, in the order of $10^{-2}$, which is consistent with a $90^{\circ}$ reorientation of the ferroelectric domains. We also demonstrated the possibility to integrate poly-BTO thin films as an upper cladding of Si waveguides with a low additional propagation loss $(<1$ $\mathrm{dB} / \mathrm{mm}$ ), enabling the realization of microring resonators and other integrated devices with the proposed polyBTO Si-photonics platform.

\section{SELF-HOLDING OPTICAL SWITCHING IN BTO THIN FILMS}

Ferroelectric materials are characterized by domains with an in-plane ( $a$-axis) or out-of-plane ( $c$-axis) orientation, which in polycrystalline films are almost randomly distributed. Domains orientation can be manipulated by applying an external electric field, exceeding the coercive field of the material. As schematically shown in Fig. 1(a), because of the domain re-orientation, a large change of the refractive index $n$ is expected. Due to the remanent polarization of ferroelectric materials, the refractive index variation is partially maintained when the electric field is turned off.

BTO films ( 100 nm thick) were grown by pulsed laser deposition (PLD) on conductive Si(001) substrates, with control of both temperature $\left(400{ }^{\circ} \mathrm{C}-600^{\circ} \mathrm{C}\right)$ and oxygen pressure $\left(p_{\mathrm{O}_{2}}=20-80 \mathrm{mT}\right.$ orr $)$ in the deposition chamber. This allowed to obtain either amorphous or polycrystalline structures. The native silicon dioxide on the surface of Si substrates was removed by standard wet etching in hydrofluoric acid prior insertion in vacuum. Moreover, the low resistivity of these substrates $(\sim 0.1 \Omega \cdot \mathrm{cm})$ allowed ferroelectric characterization and the application of electric fields across the final heterostructure, where Si can be used as back-contact. Although deposition of BTO requires oxygen pressure in the range of 20-80 mTorr for the right film stoichiometry, the first two layers were deposited at $600{ }^{\circ} \mathrm{C}$ in high-vacuum $\left(p=4 \times 10^{-7}\right.$ mbar $)$ without introducing $\mathrm{O}_{2}$ in the chamber, to avoid $\mathrm{SiO}_{2}$ formation at the interface with the substrate that would be detrimental for crystalline 
growth of the epilayer. A post-annealing at $600^{\circ} \mathrm{C}$ was then performed with a 0.5 atm $\mathrm{O}_{2}$ pressure to fill the oxygen vacancies in the BTO films.

Reflection high-energy electron diffraction (RHEED) measurements in Fig. 1(b) indicate that films deposited at $600^{\circ} \mathrm{C}$ exhibit a polycrystalline structure (poly-BTO), while lower deposition temperatures $\left(\sim 400{ }^{\circ} \mathrm{C}\right)$ favour amorphous films (a-BTO). As a confirmation, spectroscopic ellipsometry [see Fig. 1(b)] reveals that refractive index $n$ of a-BTO films $(\sim 1.9$ at $\lambda>1300 \mathrm{~nm})$ is lower than the poly-BTO films $(\sim 2.2$ at $\lambda>1300 \mathrm{~nm})$, in agreement with previous reports [1].

With the growth temperature fixed at $600^{\circ} \mathrm{C}$, the partial oxygen pressure determines the fine polycrystalline structure of poly-BTO films. X-Ray diffraction (XRD) data versus $\mathrm{O}_{2}$ partial pressure are reported in Fig. 1(c). In the film grown at $20 \mathrm{mTorr}$ (purple curve), the (001) and the (110) diffraction peaks indicate out-of-plane $c$ domains and in-plane $a$-domains, respectively. Both films grown at 40 and 20 mTorr show a coexistence of inplane and out-of-plane structural domains, whereas the one grown at 80 mTorr lacks of (001) reflection peak thus showing in-plane oriented grains. Finally, the surface roughness of poly-BTO films is between 0.7-0.9 nm rms, as measured by atomic force microscopy (AFM) in Fig. 1(d).

(a)

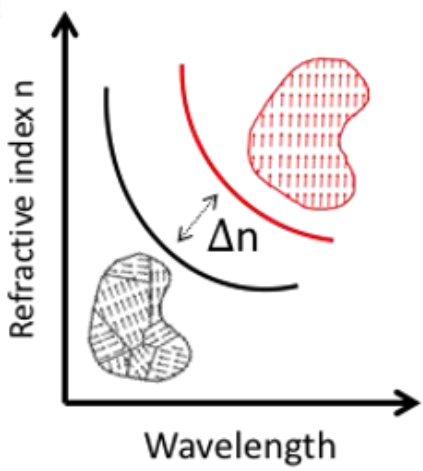

(c)

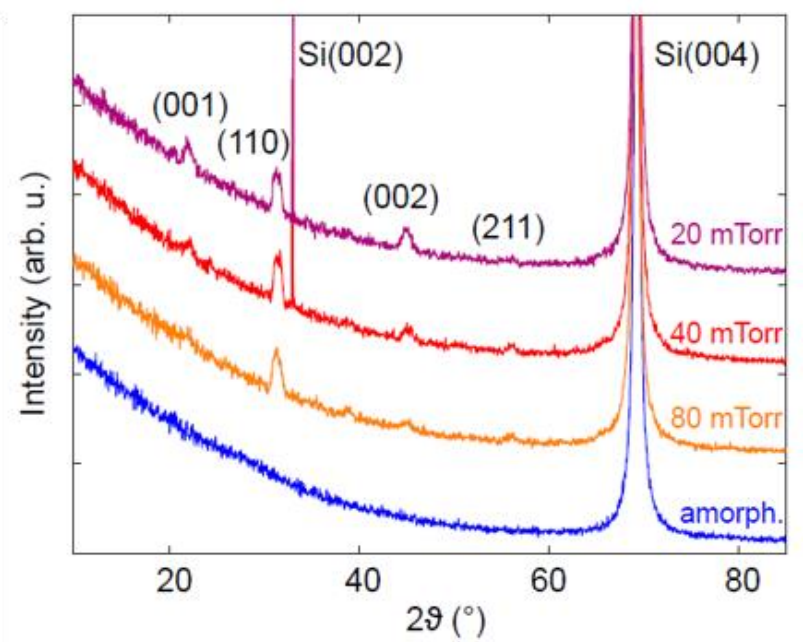

(b)

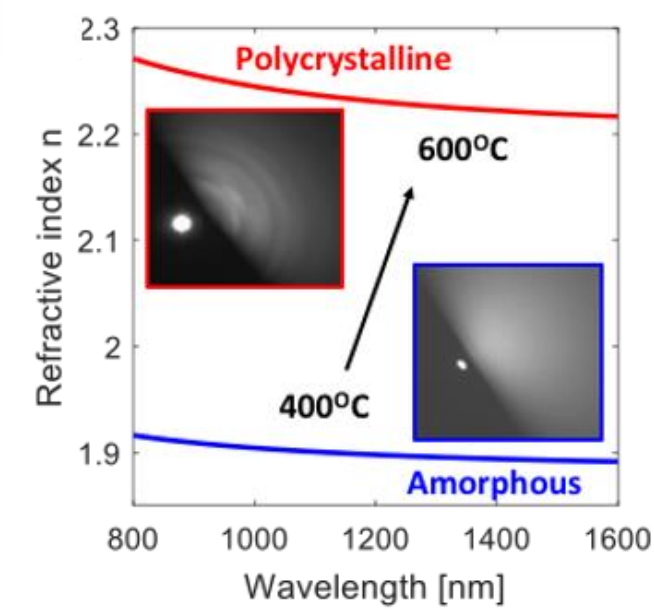

(d)

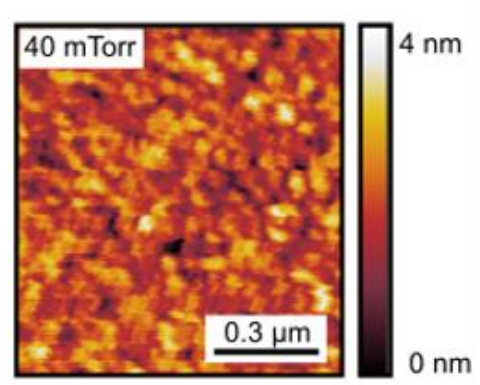

Figure 1. (a) Schematic of the refractive index change associated with domain switching in poly-BTO film. (b) Refractive index of amorphous (blue curve) and polycrystalline (red curve) BTO films deposited by PLD at different temperatures. RHEED measurements in the inset show that BTO on Si needs growth temperatures of about $600{ }^{\circ} \mathrm{C}$ to achieve crystallinity. (c) Wide range XRD $\omega$-2 $\theta$ scans versus oxygen partial pressure at fixed growing temperature of $600^{\circ} \mathrm{C}$. Peaks corresponding to different orientations of the BTO grains are visible mostly in the low oxygen pressure regime. The (001) and (002) reflections originate from out-of-plane cdomains, while the (110) reflection is associated to in-plane a-domains. Films grown at a lower temperature $\left(500{ }^{\circ} \mathrm{C}\right)$ are amorphous and do not show any XRD peak from BTO (blue curve). (d) AFM topography image gathered from poly-BTO grown with oxygen pressure of 40 mTorr. 
(a)

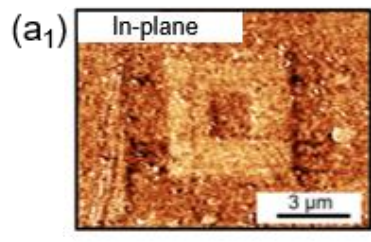

$\left(a_{3}\right)$

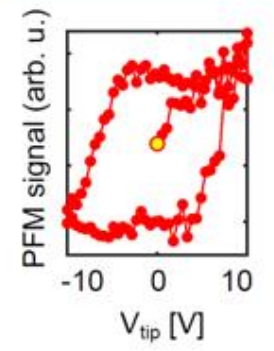

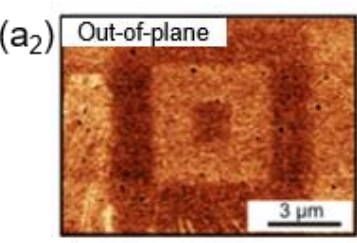

$\left(a_{4}\right)$

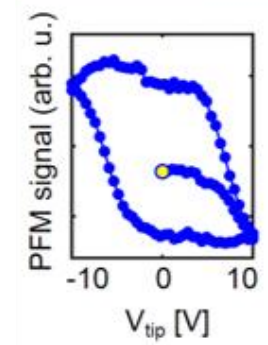

(b)

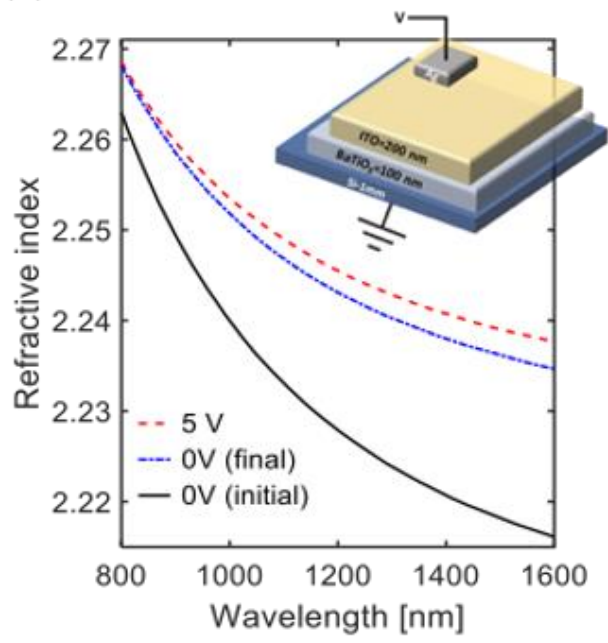

Figure 2. Ferroelectric domains measured on poly-BTO films after poling the sample with the tip at $+10 \mathrm{~V}$ and $-10 \mathrm{~V}$ over two concentric squares of 5 and $3 \mu \mathrm{m}$ per side. The in-plane $\left(a_{1}\right)$ and out-of-plane $\left(a_{2}\right)$ phase of the piezoelectric response is reported. In panels $\left(a_{3}\right)$ and $\left(a_{4}\right)$, the full ferroelectric hysteresis loops measured as PFM phase signal are reported for the in-plane and out-of-plane components of the ferroelectric polarization. (b) Measured refractive index of the poly-BTO film in the pristine state (black solid curve), during the application of a $5 \mathrm{~V}$ pulse (red dashed curve) and in the remnant state (blue dash-dotted curve).

By using piezoforce microscopy (PFM) we checked the ferroelectric properties of poly-BTO films, observing a significant PFM response for both in-plane and out-of-plane components from samples grown at 20 and 40 mTorr. PFM measurements on poly-BTO grown at 20 mTorr are shown in Fig. 2(a), containing in-plane (panels $a_{1}, a_{3}$ ) and out-of-plane (panels $a_{2}, a_{4}$ ) PFM signals. In contrast, a lower out-of-plane signal is measured in the sample grown at 80 mTorr, indicating in plane domains in agreement with XRD results of Fig. 1(c). Besides, in this sample out-of-plane domain orientation is not achievable with voltage pulses lower as high as $10 \mathrm{~V}$.

Switching experiments where performed on poly-BTO grown at 20 mTorr and $40 \mathrm{mTorr}$. A $200 \mathrm{~nm}$ layer of indium tin oxide (ITO) was sputtered onto the poly-BTO film to realize the top electrode. The change in the optical properties of the poly-BTO film was measured by spectroscopic ellipsometry before, during and after the application of an external voltage. Results resported in Fig. 2(b) show that by applying a $5 \mathrm{~V}$ signal, a large change of the refractive index of poly-BTO is induced (red dashed curve) with respect to the initial state (black solid curve), that is as large as $3 \times 10^{-2}$ in the $1550 \mathrm{~nm}$ range. Such refractive index variation, which is accompanied by no measurable change in the material loss, suggests the occurrence of some reorientation of ferroelectric domains (from $a$ to $c$ axis) in the poly-BTO film [4]. After the electric signal is switched off (blue dashed-dotted curve), the switched state was mostly preserved with only a small relaxation in the refractive index (red dashed curve). Preliminary results suggest that the pristine state of the film can be restored by heating the sample above the Curie temperature of $\mathrm{BTO}\left(120^{\circ} \mathrm{C}\right)$.

\section{INTEGRATION OF POLY-BTO FILMS IN SILICON PHOTONIC WAVEGUIDES AND CIRCUITS}

Poly-BTO was employed as upper cladding material of 220-nm thick Si nanowaveguides. As shown in the schematic of Fig. 3(a), the Si core of the waveguide was deeply etched to get a rectangular shape and was then covered with a 200-nm-thick layer of poly-BTO. To assess the loss of the poly-BTO coated waveguide, optical transmission measurements were performed on 4.5-mm-long straight waveguides with a width $w$ between 400 $\mathrm{nm}$ and $550 \mathrm{~nm}$. Figure 3(b) shows the normalized transmission before (black squares) and after (red circles) the poly-BTO upper cladding deposition. Each data point is the average transmission measured across a 60-nm-wide wavelength range centered around $1550 \mathrm{~nm}$ for transverse electric (TE) input polarization. Results show that the additional loss due to the poly-BTO film is less than $1 \mathrm{~dB} / \mathrm{mm}$ for $450 \mathrm{~nm}$ wide waveguide and $<0.2 \mathrm{~dB} / \mathrm{mm}$ for 550 -nm-wide waveguides. The loss decrease observed in the narrowest waveguide (400 $\mathrm{nm}$ width) is probably due to the mitigation of the roughness-induced scattering loss, which is less pronounced when the refractive index contrast of the waveguide reduces [5]. Similar results were also achieved for TM polarization.

Numerical simulations show that for this waveguide geometry the overlap of the guided mode with the polyBTO material is about $18 \%$ for TE polarization and $28 \%$ for TM polarization. Given the large $\Delta n$ measured on poly-BTO thin films [see Fig. 2(b)], a $\pi$-phase shift in a waveguide actuator of less than $100 \mu \mathrm{m}$ length can be 
achieved. Over such a short waveguide length, less than $0.1 \mathrm{~dB}$ loss is expected for a waveguide actuator independently of the waveguide width.

We also successfully demonstrated the possibility to employ the poly-BTO coated Si waveguide to realize integrated devices like microring resonators. Figure 3(c) shows the TE transmission measured at the drop port of a silicon microring resonator with a radius of $40 \mu \mathrm{m}$. Before the deposition of the 200-nm-thick poly-BTO upper cladding (black dashed line), the air-coated silicon microring has a free spectral range of $2.45 \mathrm{~nm}$, a $3 \mathrm{~dB}$ bandwidth of about $25 \mathrm{GHz}$, resulting in a Q factor of about 8000. After the deposition of the BTO cladding (red solid curve), the shape of the transmission spectrum is almost entirely preserved and exhibits an off-band isolation of more than $15 \mathrm{~dB}$. The reduction of the Q factor to about 6000 (33 GHz bandwidth) is essentially associated with the increase of the coupling coefficient of the microring directional coupler, which is due to the lower mode confinement in the Si core after the deposition of the poly-BTO cladding.

(a)

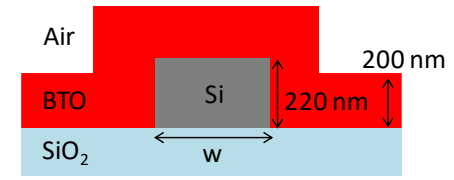

(b)

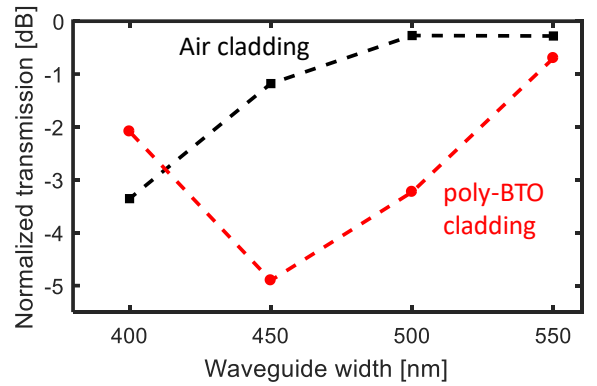

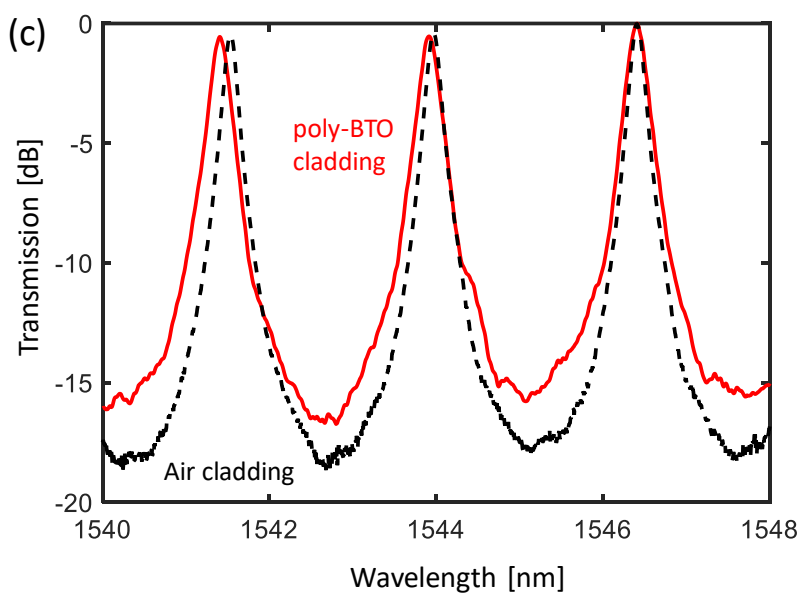

Figure 3. (a) Schematic of the cross section of a Si channel waveguide coated with a poly-BTO film. (b) Normalized transmission of 4.5-mm-long Si straight waveguides before (black squares) and after (red circles) the deposition of the poly-BTO upper cladding. (c) Normalized transmission at the drop port of a microring resonator before (black dashed curve) and after (red solid curve) the deposition of the poly-BTO upper cladding.

\section{CONCLUSIONS}

We demonstrated an electrically-driven non-volatile change of the refractive index of polycrystalline BTO films deposited by PLD, which is consistent with a partial $90^{\circ}$ reorientation of the ferroelectric domains of the material. Temperature and pressure deposition conditions to achieve a poly-BTO crystalline structure with a coexistence of in-plane and out-of-plane structural domains were investigated and the ferroelectric properties of the deposited films were assessed by PFM measurements. A large refractive index variation $\left(3 \times 10^{-2}\right)$ associated with domain reorientation was observed, which is not accompanied by any measurable loss increase. We also successfully integrated poly-BTO thin films in Si waveguides and microring resonators. Results demonstrate the feasibility of compact $(<100 \mu \mathrm{m})$, low-loss $(<0.1 \mathrm{~dB})$, self-holding phase actuators integrated in Si optical waveguides and the possibility to realize PICs with the proposed poly-BTO Si-photonics platform.

\section{ACKNOWLEDGEMENTS}

This work was supported by Fondazione Cariplo Project "Advanced Control Technologies for Integrated Optics (ACTIO)" - Rif. 2016-0881.

\section{REFERENCES}

[1] H. Zhang, et al.: Ultracompact Si-GST hybrid waveguides for nonvolatile light wave manipulation, IEEE Photonics Journal, 10(1), 2200110, 2018.

[2] A. Joushaghani, et al.: Sub-volt broadband hybrid plasmonic-vanadium dioxide switches, Applied Physics Letters 102, 061101, 2013.

[3] Thomas, Reji, D. C. Dube, M. N. Kamalasanan, and Subhas Chandra: Optical and electrical properties of BaTiO3 thin films prepared by chemical solution deposition, Thin solid films, 346 (1-2), 212, 1999.

[4] M. J. Dicken, L. A. Sweatlock, D. Pacifici, H. J. Lezec, K. Bhattacharya, and H. A. Atwater: Electrooptic modulation in thin film barium titanate plasmonic interferometers, Nanoletters, 8 (11), 4048, 2008.

[5] D. Melati, A. Melloni, and F. Morichetti: Real photonic waveguides: guiding light through imperfections, Advances in Optics and Photonics, vol. 6, no. 2, pp. 156-224, May 2014. 